\title{
High Blood Pressure in a Urea Cycle Disorder: Case Report
}

\author{
Carolina Solé, MD ${ }^{1}$ María Arriaga, $\mathrm{MD}^{1}$ Elvira Cañedo, $\mathrm{MD}^{2}$ \\ 1 Department of Neonatology, Gregorio Marañon Hospital, Madrid, \\ Spain \\ 2 Department of Metabolism Diseases and Nutrition, Niño Jesús \\ Hospital, Madrid, Spain \\ Address for correspondence Carolina Solé, MD, Neonatology \\ Department, Gregorio Marañon Hospital, O’Donnell Street, 48, 28009 \\ Madrid, Spain (e-mail: carolina.sole.delgado@gmail.com). \\ Am J Perinatol Rep 2020;10:e347-e351.
}

\begin{abstract}
Keywords

- hypertension

- citrullinemia

- arginine

- newborn

Introduction Urea cycle disorders (UCDs) form a group of metabolic pathological conditions that might develop serious neurological consequences. Early diagnosis, before irreversible damage is established, is the most important prognostic and morbidity factor.

Case Report We present the case of a 5-day newborn with high blood pressure and respiratory distress. Diagnosis was type I citrullinemia. With appropriate citrullinemia guided-treatment blood pressure returned to normal.

Conclusion High blood pressure has been rarely described as a lead symptom for the debut of a UCD. We must take this into consideration as an early recognition and treatment of these disorders are of the utmost importance.
\end{abstract}

\section{Case Report}

Our patient is a 5-day male newborn, with no pathological clinical record and no parental consanguinity presenting in the emergency department for low volume urine output in the last 12 hours. Parents referred no other symptoms. On the physical examination it stands out respiratory distress and maintained high blood pressure (BP) in the four limbs (above 120/90 $\mathrm{mm} \mathrm{Hg}$ ). Heart rate, peripheral oxygen saturation $\left(\mathrm{SatO}_{2}\right)$, and temperature were within normal range. Full blood count, renal and liver function, C-reactive protein (CRP), venous blood gas, and urine sediment were examined and found normal except for a respiratory alkalosis. Also, urine and blood culture were taken, and empirical antibiotic treatment started. He was transferred to a tertiary care neonatal center to continue study and support.

On arrival to our center, he was ill with continuous respiratory moan and work of breathing. He also had an altered mental status of being lethargic. No other findings or dysmorphic features were observed. We corroborated the high BP both in arms and legs (respiratory rate of $50 \mathrm{rpm}$, and $\mathrm{SatO}_{2} 98 \%$ ).

Some additional tests were added, showing mild coagulopathy, an ammonia of $93 \mu \mathrm{mol} / \mathrm{L}$, and slight left ventricular hypertrophy. Cranial and abdominal ultrasound were normal as the CRP for enterovirus, cytomegalovirus, herpes 1,2 , zoster, and parechovirus.

After a few hours of admission, the metabolic screening center informs of an altered result for the patient (sample on dry paper at 48 hours of life) with elevated citrulline and normal arginosuccinate acid, suggesting urea cycle disorder (UCD), specifically type I citrullinemia as we can see in -Table 1. At that time ammonia levels had risen to 243 $\mu \mathrm{mol} / \mathrm{L}$. Another plasma and urine sample were collected for amino acid analysis; (results in - Table 2 ) then, treatment of type I citrullinemia-oriented case was initiated with sodium benzoate, sodium phenylbutyrate, L-arginine, and carnitine. Calory intake was maintained with glucose and lipids, with protein restriction. Despite the initiation of the treatment, ammonia quickly rose to a maximum of $875 \mu \mathrm{mol} / \mathrm{L}$ in the first 12 hours, requiring venous hemofiltration. After this, it was finally possible to control hyperammonemia.

The patient needed intensive care with mechanical ventilation, inotropic support, and hemofiltration for 3 days. Blood pressure normalized within 8 hours of the treatment.

From a neurological point of view: at admission, he was hypoactive with both limited range of movement and response received

June 11,2020

accepted

June 15, 2020
DOI https://doi.org/

10.1055/s-0040-1716733. ISSN 2157-6998.
Copyright $\odot 2020$ by Thieme Medical Publishers, Inc., 333 Seventh Avenue, New York, NY 10001, USA. Tel: +1(212) 760-0888.
License terms

()(1) $\Theta \circledast$ 
e348 High Blood Pressure in a Urea Cycle Disorder Delgado et al.

Table 1 Results of metabolic screening $48 \mathrm{~h}$ of life

\begin{tabular}{|l|l|l|}
\hline Amino acids & $\mu \mathrm{mol} / \mathrm{L}$ & $\begin{array}{l}\text { Reference interval } \\
\text { for 48 } \mathrm{h} \text { of life }\end{array}$ \\
\hline Alanine & 327 & $124.32-419.24$ \\
\hline Arginine & 9.53 & $2.73-41.07$ \\
\hline Citrulline & 1010 & $5.21-27.07$ \\
\hline Glutamine + lysine & 1760 & $363.83-1145.86$ \\
\hline Glutámic acid & 306 & $121.93-464.41$ \\
\hline Glicine & 409 & $227.47-752.40$ \\
\hline $\begin{array}{l}\text { Leucine + isoleucine }+ \\
\text { hydroxyproline }\end{array}$ & 117 & $53.78-206.34$ \\
\hline Methionine & 46.4 & $8.52-30.73$ \\
\hline Ornithine & 39.6 & $33.30-180.62$ \\
\hline Phenylalanine & 65.5 & $25.34-75.42$ \\
\hline Proline & 189 & $68.10-219.03$ \\
\hline Succinylacetone & 0.22 & $0.07-0.54$ \\
\hline Tyrosine & 115 & $27.47-187.58$ \\
\hline Valine & 107 & $49.55-232.38$ \\
\hline Argininosuccínic acid & 0.12 & $0.08-0.48$ \\
\hline
\end{tabular}

Table 2 Amino acid profile at $5 \mathrm{~d}$ of age

\begin{tabular}{|l|l|l|}
\hline Amino acids & $\mu \mathrm{mol} / \mathrm{L}$ & Normal values \\
\hline Arginine & 9 & $61 \pm 27$ \\
\hline Citrulline & 4,002 & $19 \pm 9$ \\
\hline Glutamine & $2,197^{\mathrm{a}}$ & $506 \pm 135$ \\
\hline Lysine & 438 & $154 \pm 55$ \\
\hline Methionine & $347^{\mathrm{a}}$ & $27 \pm 9$ \\
\hline Ornithine & 14 & $79 \pm 37$ \\
\hline
\end{tabular}

to stimulus. Altered consciousness, rapidly evolved from stupor to drowsiness. Abnormal amplitude integrated electroencephalography (aEEG) with a burst suppression pattern. On the second day, he presented with clinical seizures of low amplitude clonisms of the head with acute increase work of breathing. It was necessary to provide phenobarbital and levetiracetam bolus to control seizures, maintaining levetiracetam as a prophylactic treatment. Significant clinical improvement was noticed on the third day of admission, with continuous pattern on the aEEG, normal awareness level, appropriate eye tracking, adequate peripheral and axial tone, present both osteotendinous and archaic reflexes, harmonious and symmetrical movement of the four limbs and normal strength. He had normal EEG. Brain magnetic resonance imaging showed altered signal on basal ganglia with hyperintensity in T1 of nucleus pallidus and hypointensity on white matter with punctiform hyperintensity lesions. Findings in line with $U C D^{1}$ are shown in - Figs. 1 to $\mathbf{5}$.

After control of HA, low protein intake $(0.3 \mathrm{~g} / \mathrm{kg} / \mathrm{d})$ was started with progressive increase of dose up to $1.6 \mathrm{~g} / \mathrm{kg} / \mathrm{d}$. Well-tolerated and daily ammonia check was normal. Intravenous medication was changed to enteral L-arginine (350 mg/ $\mathrm{kg} / \mathrm{d}$ ), carnitine (30 mg/kg/d), and glycerol phenylbutyrate. He

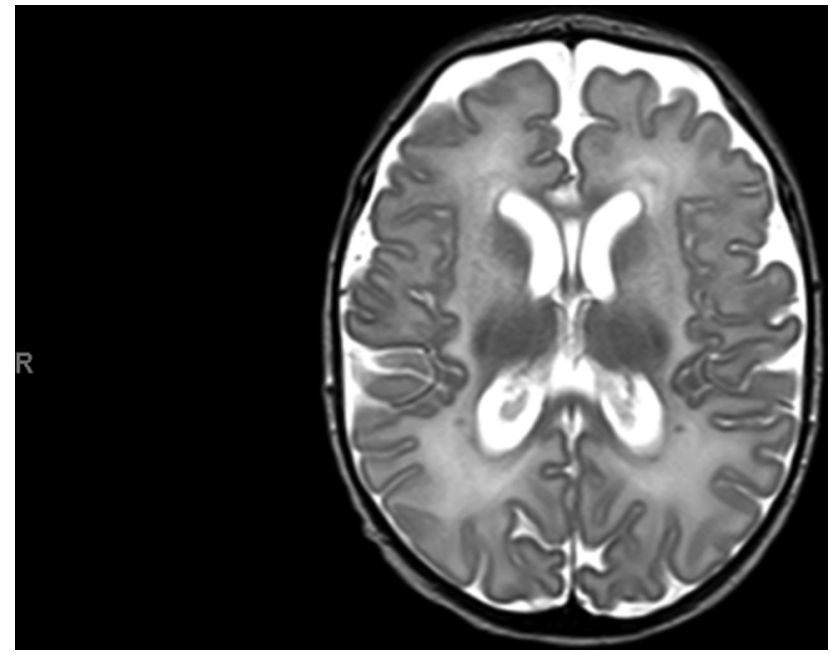

Fig. 1 Axial T2-weighted image showing high intensity alteration on white matter with smaller low intensity signals.

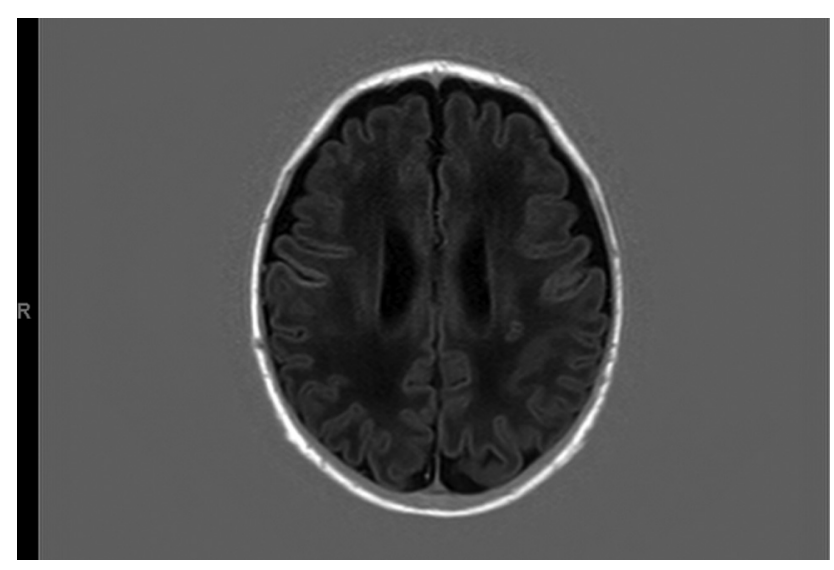

Fig. 2 T1-weighted low intensity signal on white matter and focal high intensity signal.

was discharged home with multidisciplinary follow-up with pending results of the genetic study.

\section{Discussion}

The urea cycle is a metabolic pathway that transforms ammonia into urea. The result of the catabolism of amino acids is ammonia, a high toxic compound that cannot be excreted unless it is processed into a high solubility substance such as urea. Genetic defects in the enzymes involved in the cycle can occur, the result is a UCD. These can be ${ }^{2}$ :

- $N$-Acetyl glutamate synthase deficiency (NAGS; OMIM 237310).

- Carbamoyl phosphate synthetase deficiency (CPS-1; OMIM 237300).

- Ornithine transcarbamoylase deficiency (OTC; OMIM 311250).

- Citrullinemia type I (deficiency of argininosuccinate acid synthase [ASS]; OMIM 215700).

- Argininosuccinic aciduria (deficiency of argininosuccinate acid lyase [ASL]; OMIM 207900).

- Argininemia (deficiency of arginase [ARG]; OMIM 207800). 

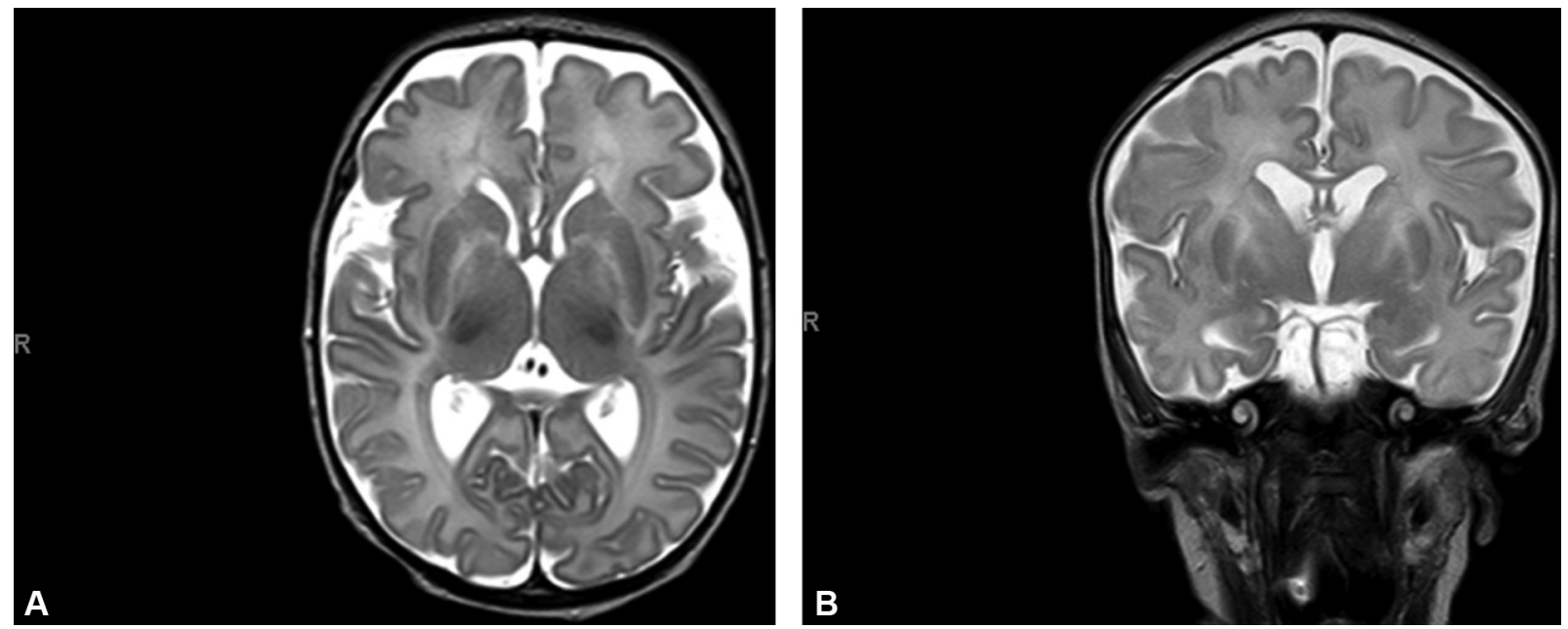

Fig. 3 (A and B) Axial and coronal, T2-weighted image showing low intensity of globi pallidi.
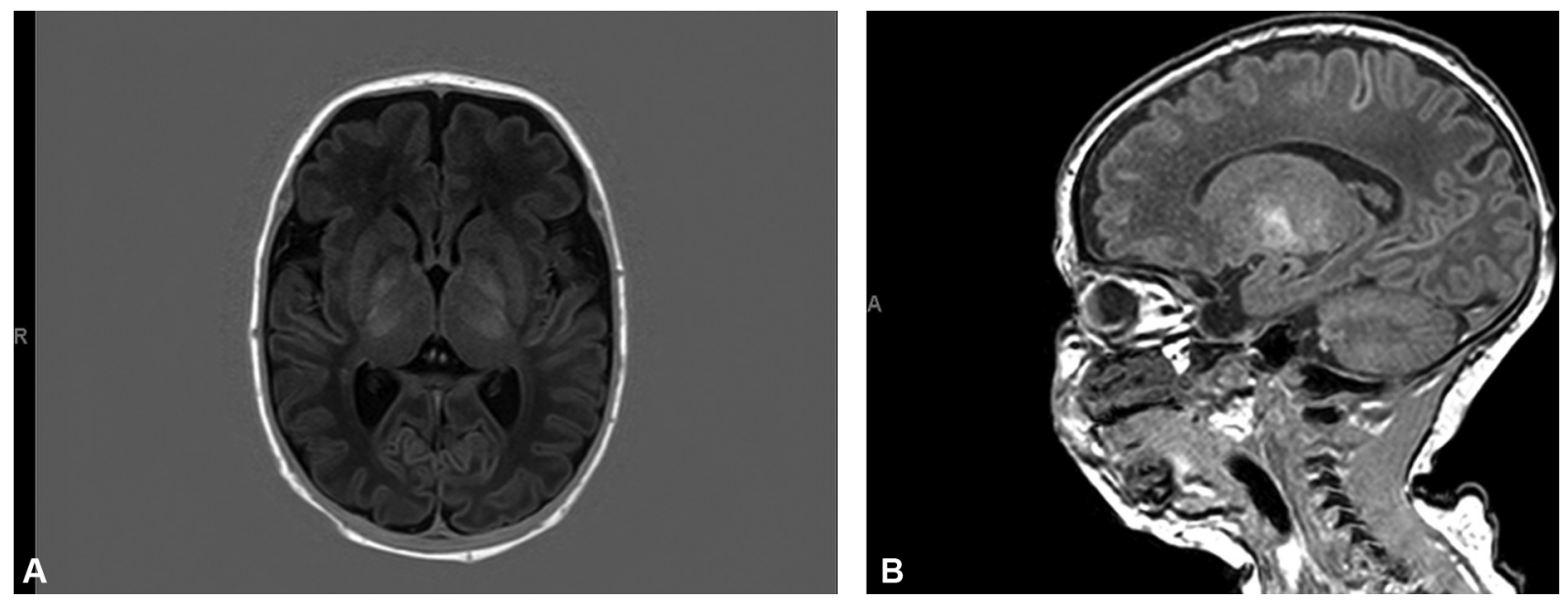

Fig. 4 (A and B) Axial and sagittal, T1-weighted high intensity signal of globi pallidi.
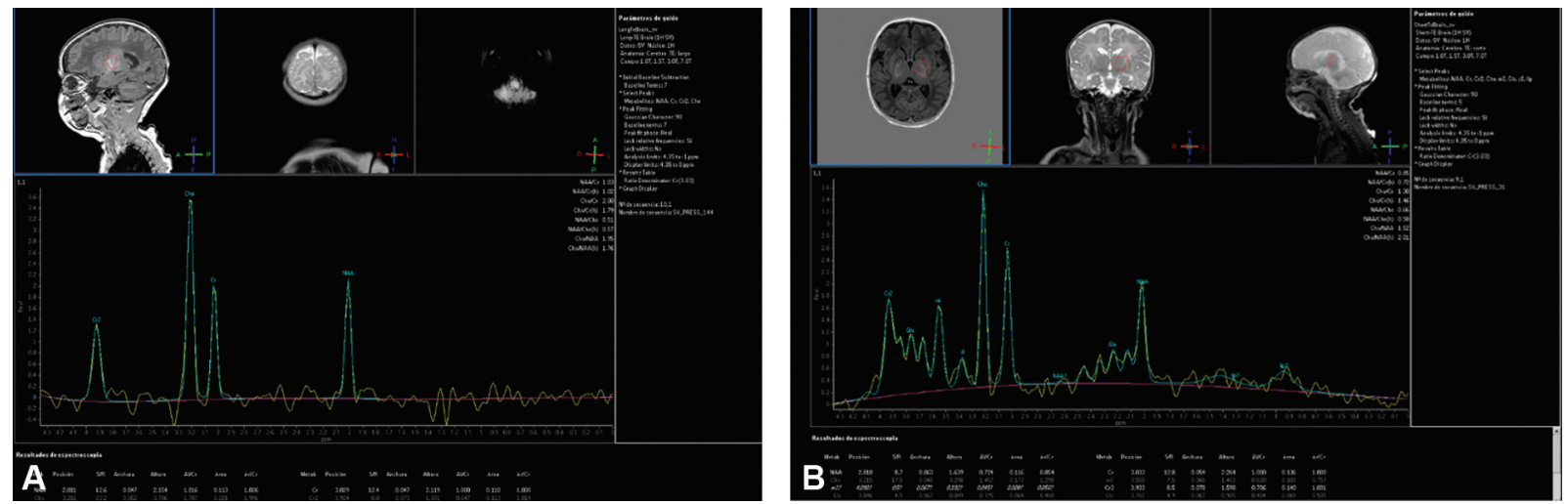

Fig. 5 (A and B) Normal MR spectroscopy. MR, magnetic resonance.

We can include three deficiencies of membrane transporters:

- Hyperornithinemia, hyperammonemia, homocitrullinuria syndrome (deficiency of the mitochondrial ornithine transporter).

- Lysinuria with protein intolerance (due to altered transport of dibasic amino acids).
- Citrine deficiency or citrullinemia type 2 (aspartame-glutamate mitochondrial hepatic transporter deficit; - Fig. 6).

In this case, given the increase in citrulline and the decrease in arginosuccinate, the diagnostic suspicion is an alteration in arginine synthetase and therefore a type I citrullinemia. Even so, regardless of the suspected diagnosis, the main acute manifestations of the UCD are those 


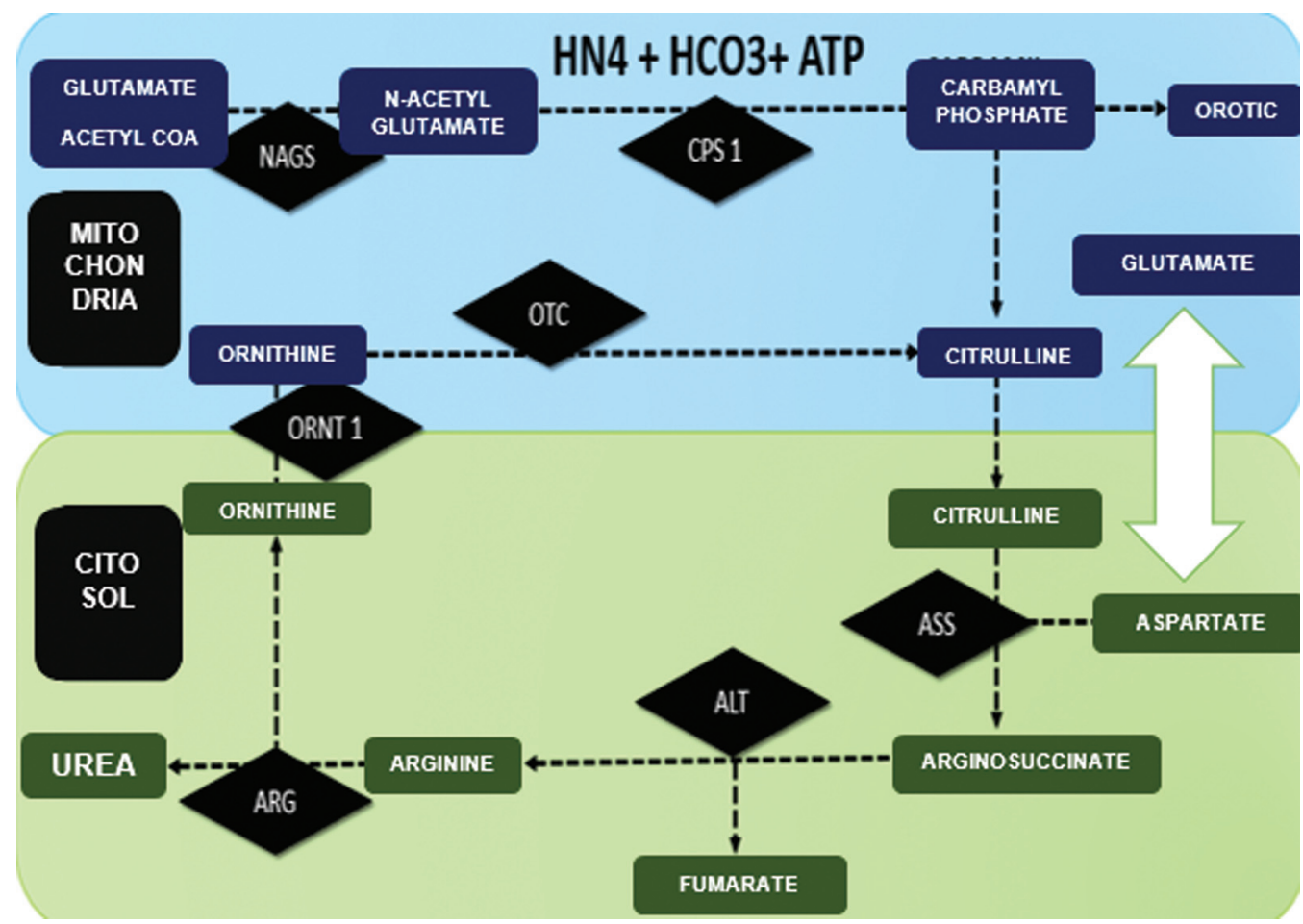

Fig. 6 Urea cycle in the liver. Ammonia reacts with bicarbonate and with $N$-acetyl glutamate to produce carbamoyl phosphate (CPS). The CPS yields its carbamoyl group to ornithine to form citrulline. Citrulline is released to cytoplasm. The second amyl group from aspartate is condensed with citrulline to form arginosuccinate, which is hydrolyzed into free arginine and fumarate. The fumarate enters the Krebs cycle and arginine hydrolyzes to form urea (excreted in the urine) and ornithine, which re-enters the mitochondria to restart the cycle. Mitochondrial enzymes (upper part of the cycle): $N$-acetyl glutamate synthetase (NACS) essential co-activator of CPS-1; carbamoyl phosphate synthetase (CPS-1); Ornithine transcarbamylase (OTC). Cytoplasmatic enzymes (or lower part of the cycle): arginosuccinate synthetase (ASS); arginosuccinate lyase (ASL); arginase (ARG). Ornithine transporter deficit (ORNT-a) produces HHH syndrome. $\mathrm{HHH}$, hyperornithinemia, hyperammonemia, homocitrullinuria.

derived from hyperammonemia (HA). Ammonia is a highly toxic product, especially on the central nervous system. It is considered as a potentially lethal metabolic emergency. The clinical syndrome of the newborn (which is the form of debut in $60 \%$ of the cases) begins at 24 to 72 hours of life, producing decrease feeds, thermic dysregulation, vomiting, impaired level of consciousness (drowsiness that evolves into coma), and seizures. A common sign is central hyperventilation and respiratory alkalosis. This may evolve to respiratory and cardiac failure.

An ammonia level $>110 \mathrm{mmol} / \mathrm{L}$ in the neonatal period is considered pathological. In the case of suspicion, therapeutic measures should be initiated immediately. ${ }^{3}$ In our case, these measures were aimed at the diagnosis of suspected type 1 citrullinemia which does not respond to cofactors or carglumic acid (Carbaglu).

\section{Why High Blood Pressure?}

The patient debuted with arterial hypertension as a lead symptom. Hypertension screening was performed:

- Heart disease: there were no differences in BP or $\mathrm{SatO}_{2}$ between upper or lower limbs. Chest X-ray without cardiomegaly or acute lung edema was obtained. An emergency echocardiogram was performed showing only a concentric hypertrophy of the left ventricle.
- Acute renal failure, renal stenosis: Had normal renal function. Doppler ultrasound of the renal veins was performed without alterations.

- Intracranial hypertension/acute bleeding: normal heart rate. Normotensive fontanelle.

- Cranial ultrasound was normal.

- Endogenous production of catecholamines: abdominal ultrasound without masses. Normal urine metanephrines.

After the diagnosis of suspected type I citrullinemia, we reviewed the pathophysiology and previous literature. This disorder involves arginine deficiency. It cannot be synthesized and forms an essential part of amino acid, as can be seen in - Fig. 7. L-arginine acts as a substrate for nitric oxide synthetase to form endothelial nitric oxide and citrulline. ${ }^{4}$

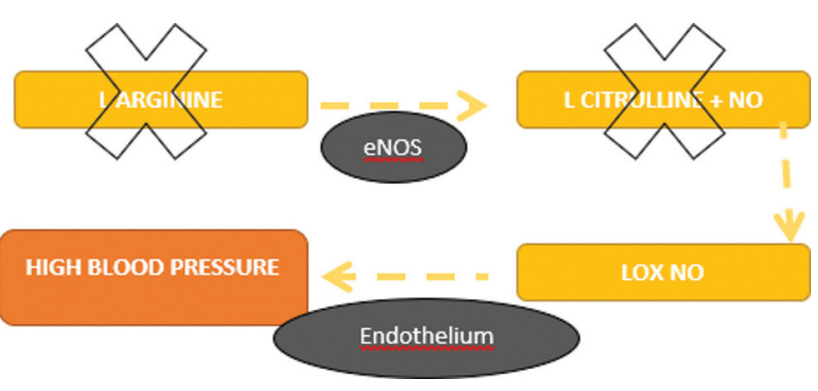

Fig. 7 L-arginine acts as a substrate for nitric oxide synthetase (ONS) to form endothelial nitric oxide (NO) and citrulline. 
A reduction in NO production plays a major role in vascular tone and hence increase in $\mathrm{BP} .{ }^{4,5}$ As it can be seen in - Table 1, arginine levels at 48 hours of life were in the lower reference value. At that moment, our patient was asymptomatic. At 5 days of age, when he debuted with hypertension, arginine was below normal range. Once treatment was started, which included arginine supplementation, the patient's BP normalized. The connection between hypertension and UCD had barely been described, with little representation in this specific literature. Recently, Teufel et $\mathrm{al}^{6}$ described a series of 17 patients in which $81 \%$ of them presented with BP $>95$ th percentile at debut. They also, highlighted how this clinical parameter could be useful to discern a UCD debut from a neonatal sepsis. Although the study involves a small series of patients, it underlines and supports what we have seen in this particular case and the importance of having present UCD as a differential diagnosis in a newborn with high BP.

\section{Conclusion}

Hypertension and UCD maintain a clear pathophysiological relationship, barely described in the literature, which can prove to be very useful in daily clinical practice.

\section{Authors' Contribution}

C.S.D. wrote the manuscript. M.A.R. and E.C. reviewed and corrected the manuscript. All authors approved the final version.

\section{Conflict of Interest}

C.S.D., M.A.R., and E.C. declare that they do not have a conflict of interest. Informed written consent was obtained from parents of the patient.

\section{References}

1 Takanashi J, Barkovich AJ, Cheng SF, et al. Brain MR imaging in neonatal hyperammonemic encephalopathy resulting from proximal urea cycle disorders. AJNR Am J Neuroradiol 2003;24(06): 1184-1187

2 Summar ML, Mew NA. Inborn errors of metabolism with hyperammonemia: urea cycle defects and related disorders. Pediatr Clin North Am 2018;65(02):231-246

3 Häberle J, Burlina A, Chakrapani A, et al. Suggested guidelines for the diagnosis and management of urea cycle disorders: first revision. J Inherit Metab Dis 2019;42(06):1192-1230. Doi: 10.1002/ jimd. 12100

4 Khalaf D, Krüger M, Wehland M, Infanger M, Grimm D. the effects of oral l-arginine and l-citrulline supplementation on blood pressure. Nutrients 2019;11(07):1679

5 Brunetti-Pierri N, Erez A, Shchelochkov O, Craigen W, Lee B. Systemic hypertension in two patients with ASL deficiency: a result of nitric oxide deficiency? Mol Genet Metab 2009;98(1-2): 195-197

6 Teufel U, Burgard P, Meyburg J, et al. High blood pressure, a red flag for the neonatal manifestation of urea cycle disorders. Orphanet J Rare Dis 2019;14(01):80 